\title{
Tobacco MAP Kinase Phosphatase (NtMKP1) Negatively Regulates Wound Response and Induced Resistance Against Necrotrophic Pathogens and Lepidopteran Herbivores
}

\author{
Kumiko Oka, ${ }^{1}$ Yuta Amano, ${ }^{2}$ Shinpei Katou, ${ }^{3}$ Shigemi Seo, ${ }^{1}$ Kei Kawazu, ${ }^{4}$ Atsushi Mochizuki, ${ }^{4}$ \\ Kazuyuki Kuchitsu, ${ }^{2}$ and Ichiro Mitsuhara ${ }^{1}$ \\ ${ }^{1}$ Plant-Microbe Interactions Research Unit, National Institute of Agrobiological Sciences, Tsukuba, Ibaraki 305-8602, Japan; \\ ${ }^{2}$ Department of Applied Biological Science, Tokyo University of Science, Noda, Chiba 278-8510, Japan; ${ }^{3}$ International Young \\ Researchers Empowerment Center, Shinshu University, Minamiminowa, Nagano 399-4598, Japan; ${ }^{4}$ National institute for \\ Agro-Environmental Sciences, Tsukuba, Ibaraki 305-8604, Japan
}

Submitted 22 November 2012. Accepted 11 February 2013.

Mitogen-activated protein kinase (MAPK) cascades are universal signal transduction pathways in eukaryotic cells. In tobacco, two MAPK, wound-induced protein kinase (WIPK) and salicylic acid (SA)-induced protein kinase (SIPK), are activated by biotic and abiotic stresses. Both WIPK and SIPK positively regulate the biosynthesis of jasmonic acid (JA) or ethylene (ET) while negatively regulating SA accumulation. We showed previously that recombinant tobacco MAPK phosphatase (NtMKP1) protein dephosphorylates and inactivates SIPK in vitro, and overexpression of NtMKP1 repressed wound-induced activation of both SIPK and WIPK. To elucidate the role of NtMKP1 in response to biotic and abiotic stresses, we generated transgenic tobacco plants in which $N$ tMKP1 expression was suppressed. Suppression of NtMKP1 expression resulted in enhanced activation of WIPK and SIPK and production of both JA and ET upon wounding. Wound-induced expression of JA- or ET-inducible genes, basic PR-1 and PI-II, was also significantly enhanced in these plants. Furthermore, NtMKP1-suppressed plants exhibited enhanced resistance against a necrotrophic pathogen, Botrytis cinerea, and lepidopteran herbivores, Mamestra brassicae and Spodoptera litura. These results suggest that NtMKP1 negatively regulates wound response and resistance against both necrotrophic pathogens and herbivorous insects through suppression of JA or ET pathways via inactivation of MAPK.

Mitogen-activated protein kinases (MAPK) are well conserved in all eukaryotes and play a central role in transducing extracellular stimuli to intracellular responses (MAPK group 2002; Zhang and Liu 2001). MAPK are implicated in regulating growth, development, and responses to environmental stress in plants (Pitzschke et al. 2009). In response to a wide

K. Kawazu and A. Mochizuki contributed equally to this work.

Corresponding author: I. Mitsuhara; E-mail: mituhara@ affrc.go.jp

* The $\boldsymbol{e}$-Xtra logo stands for "electronic extra" and indicates that three supplementary figures are published online.

(C) 2013 The American Phytopathological Society range of biotic and abiotic stresses, MAPK are activated via phosphorylation by upstream MAPK kinases (MAPKK) which, in turn, are activated by upstream MAPKK kinases (MAPK Group 2002; Nakagami et al. 2005).

In tobacco (Nicotiana tabacum), two MAPK-woundinduced protein kinase (WIPK) (Seo et al. 1995) and salicylic acid (SA)-induced protein kinase (SIPK) (Zhang and Klessig 1997) — are activated by various stresses such as wounding, pathogen infection, and herbivore attack (Zhang and Klessig 2001). These MAPK are activated within a few minutes after wounding, preceding the subsequent accumulation of jasmonic acid (JA) (Seo et al. 2007). We have previously indicated that transgenic tobacco plants in which expression of SIPK and WIPK genes was suppressed by RNA silencing exhibited reduced accumulation of JA and ethylene (ET) but enhanced accumulation of SA in response to wounding and Tobacco mosaic virus (TMV) infection (Kobayashi et al. 2010; Seo et al. 2007). These results suggest that WIPK and SIPK positively regulate stress-induced accumulation of JA and negatively regulate accumulation of SA (Seo et al. 2007). The contribution of several orthologs of WIPK and SIPK upon induction of JA accumulation after wounding has been reported in other plant species (Kandoth et al. 2007; Wu et al. 2007).

JA, ET, and SA play important roles in plant defense response to pathogens and herbivores. In general, JA induces plant resistance against necrotrophic pathogens and herbivores, especially lepidopteran herbivores, whereas SA induces resistance against biotrophic pathogens (Browse 2009; Glazebrook 2005; Hind et al. 2011; Howe and Jander 2008). Wound signaling also triggers defense mechanisms that are effective against pathogens and herbivores through the regulation of these compounds (Bostock 1999; Turner et al. 2002). From those previous reports in Arabidopsis, tomato, and N. attenuata (Diaz et al. 2002; Glazebrook 2005; Koornneef and Pieterse 2008; Meldau et al. 2009; Wu et al. 2007), it surmises that tobacco MAPK may modulate resistance to necrotrophic pathogens and herbivores through defenserelated signal cross talk between JA or ET and SA, which positively regulating biosynthesis or accumulation of JA or ET while negatively regulating the accumulation of SA.

MAPK are negatively regulated by dephosphorylation through Ser/Thr phosphatases (PP2C family), Tyr phosphatases (PTP), or dual-specificity phosphatases (DSP), known as MAPK phosphatases (MKP), through specific dephosphorylation of 
both phosphoserine/phosphothreonine and phosphotyrosine residues (Camps et al. 2000; Kerk et al. 2002; Schweighofer et al. 2007). Each Arabidopsis MKP (AtMKP), including the previously identified AtMKP1, AtMKP2, AtDsPTP1, IBR5, PHS1, and AP2C1 in Arabidopsis, is considered to be responsible for the modulation of 20 potential MAPK targets (Kerk et al. 2002; Lee and Ellis 2007; Schweighofer et al. 2007). AtMKP1 interacts with MPK6, an ortholog of tobacco SIPK, and works as a repressor of SA synthesis through MPK3/MPK6 (Anderson et al. 2011; Bartels et al. 2009; Ulm et al. 2002). IBR5 and PHS1 have been shown to regulate MPK12 and MPK18 (Lee et al. 2009; Walia et al. 2009). AtDsPTP1 and AtMKP2 dephosphorylate MPK4 and MPK3/MPK6, respectively (Gupta et al. 1998; Lee and Ellis 2007; Lumbreras et al. 2010). AP2C1 of the PP2C family interacted with MPK4 and MPK6 (Schweighofer et al. 2007). Mutations of the AtMKP1, AtMKP2, IBR5, and PHS1 genes cause higher sensitivity to genotoxic and salt stresses, hypersensitivity to oxidative stresses, reduced responses to the phytohormones auxin and abscisic acid, and compromised cortical microtubule functions, respectively (Lee and Ellis 2007; Monroe-Augustus et al. 2003; Naoi and Hashimoto 2004; Ulm et al. 2001, 2002). Moreover, Anderson and associates (2011) demonstrated that AtMKP1 plays a role for a negative regulator of AtMKP6-mediated plant defense responses. We also reported that the rice (Oryza sativa) MKP1 (OsMKP1) negatively regulates rice wound responses through the regulation of rice MAPK OsMPK3 and OsMPK6 (Katou et al. 2007).

We showed previously that a tobacco ortholog of AtMKP1, NtMKP1, dephosphorylates and inactivates SIPK in vitro
(Katou et al. 2005; Yamakawa et al. 2004). NtMKP1 has four characteristic domains: a dual-specificity phosphatase catalytic domain, a gelsolin homology domain, a calmodulin-binding domain, and a C-terminal domain (Yamakawa et al. 2004). Deletion analysis revealed that the $\mathrm{N}$-terminal noncatalytic region of NtMKP1 bound SIPK and was essential for inactivating SIPK (Katou et al. 2005). In transgenic tobacco plants overexpressing NtMKP1, the activation of both WIPK and SIPK and JA production after wounding was suppressed, whereas SA production was abnormally enhanced (Seo et al. 2007; Yamakawa et al. 2004). These results suggest that NtMKP1 may negatively regulate WIPK and SIPK; however, a knockdown study is required to evaluate the contribution of NtMPK1 to the regulation of defense signaling upon biotic and abiotic stresses.

In this study, we produced NtMKPl-suppressed tobacco plants by introducing an antisense construct. NtMKPl-suppressed plants exhibited enhanced wound response and resistance against necrotrophic pathogen and lepidopteran herbivores, indicating that NtMKP1 acts as a negative regulator of WIPK- and SIPK-mediated induced resistance in tobacco plants.

\section{RESULTS}

\section{Generation of NtMKP1-suppressed tobacco plants.}

Transgenic tobacco plants in which NtMKPl was silenced were produced by introducing an NtMKPl antisense construct under the control of an enhanced 35S promoter via Agrobacterium-mediated transformation. More than 10 independent trans-
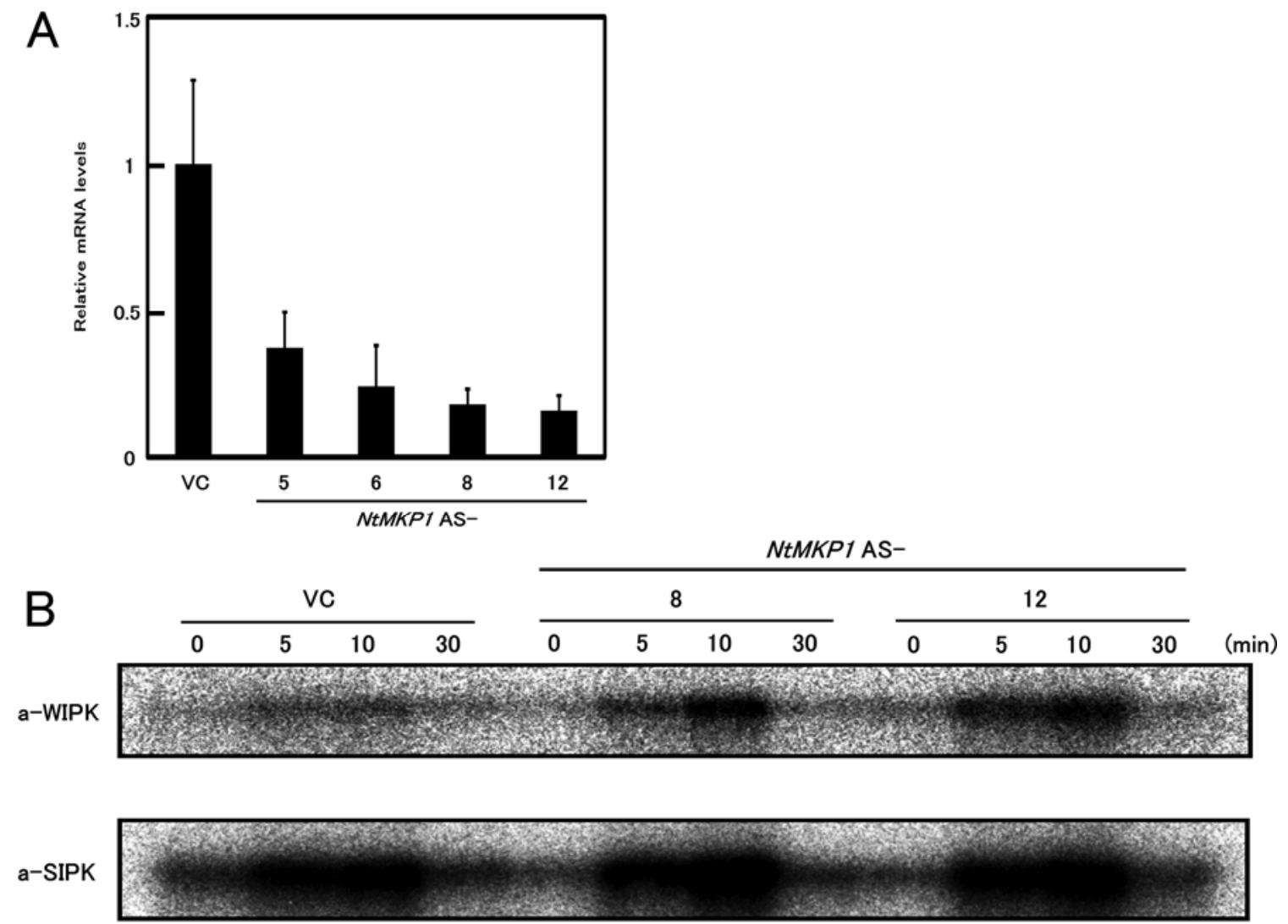

Fig. 1. Analysis of $N t M K P 1$-suppressed tobacco plants. A, Levels of transcript for NtMKP1 in vector control (VC) and four NtMKP1-AS lines. Total RNA was extracted from leaves of four representatives of transgenic tobacco lines in which NtMKP1 was introduced in the antisense orientation, and the transcript levels of $N t M K P 1$ were determined by quantitative real-time polymerase chain reaction, normalized by the level of actin as an internal standard, and shown as a value relative to that of the VC. Data represent the means \pm standard error of three independent plants for VC and NtMKP1-AS lines. B, Effect of NtMKP1 suppression on wound-induced activation of wound-induced protein kinase (WIPK) and salicylic-acid-induced protein kinase (SIPK). Total proteins were extracted from leaves of VC and two NtMKP1-AS lines before and after wounding. Kinase activities of WIPK and SIPK were determined by an immunocomplex kinase assay using myelin basic protein (MBP) as a substrate. For A and B, a transgenic tobacco plant transformed by empty vector was used as the control line. 
genic plants were obtained and levels of mRNA for NtMKPl in leaves of four representative transgenic lines in the second generation were analyzed. Quantitative real-time polymerase chain reaction (PCR) analysis revealed that the transcript levels of NtMKPI in all transgenic lines were reduced substantially compared with the control line (Fig. 1A). Two transgenic lines (NtMKP1 AS8 and AS12), which showed the lowest of NtMKP1 transcript accumulation levels, were selected for the further analysis. There were no observable differences between all transgenic lines and wild-type plants under normal growth conditions.

Next, the activities of WIPK and SIPK in NtMKPl-suppressed (NtMKPl-AS) lines were compared with those in the control line. Enhanced activation of both WIPK and SIPK by wounding was detected in two NtMKPl-AS lines, while the pattern of activation of both was similar to those in the control line (Fig. 1B). Levels of transcripts for WIPK and SIPK determined by quantitative real-time PCR analysis were not different between NtMKPI-AS lines and the control line (Supplementary Fig. S1). Thus, the reduced levels of NtMKP1 transcripts may result in insufficiency of NtMKP1 activity leading to enhanced activity of these MAPK in response to wounding.

Effect of NtMKP1 suppression on wound-induced responses.

Wound-induced accumulation of JA and ET emission was analyzed in NtMKPl-AS lines in which wound-induced WIPK and SIPK activity was enhanced. There was no significant difference in JA levels before wounding in two NtMKP1-AS and control lines. However, wound-induced JA accumulation in NtMKP1 AS8 and AS12 lines was 2.5-fold and 1.5-fold (Mann-Whitney's $u$ test, $P<0.05$ ) higher, respectively, than in the control line (Fig. 2A). Wound-induced ET emission in two NtMKP1-AS lines was also increased twice (Mann-Whitney's $u$ test, $P<0.05$ ) that of the control line (Fig. $2 \mathrm{~B}$ ). We next examined the effect of the suppression of $N t M K P 1$ on SA accumulation. TMV-inoculated leaf discs from NtMKPl-AS8 and the control line were incubated at $30^{\circ} \mathrm{C}$ for $40 \mathrm{~h}$ and transferred to $20^{\circ} \mathrm{C}$, and the endogenous levels of SA were deter- mined $8 \mathrm{~h}$ after the temperature shift. The levels of SA and a glucose conjugate of SA (SAG) were not different between NtMKP1-AS8 and the control line both before and after the inoculation of TMV (Supplementary Fig. S2).

The expression of tobacco defense-related genes in two NtMKPl-AS lines and a control line from 0 to $48 \mathrm{~h}$ after wounding was analyzed by quantitative real-time PCR. The expression levels of all genes were the highest at $24 \mathrm{~h}$ after wounding. Wound-induced accumulation of transcripts for JAdependent wound-responsive genes basic $P R-1$ and PI-II (Brederode et al. 1991; Niki et al. 1998) was significantly higher in two NtMKPl-AS lines than those in the control line (Student's $t$ test, $P<0.05$ or 0.01 ) (Fig. 3). The expression levels of acidic PR-1 and acidic PR-2 genes, which are inducible by SA (Brederode et al. 1991; Niki et al. 1998), were not altered significantly (Fig. 3). These results suggest that NtMKP1 acts as a negative regulator of tobacco wound responses.

\section{Role of NtMKP1 in resistance against necrotrophic pathogens and lepidopteran herbivores.}

NtMKP I-AS tobacco lines exhibited enhanced production of JA and ET, which are important plant signal molecules for resistance against necrotrophic pathogens and herbivores. Therefore, we evaluated the effect of NtMKPl suppression upon growth of the necrotrophic fungal pathogen Botrytis cinerea. Detached tobacco leaves from each line were inoculated with a culture of $B$. cinerea mycelium and the diameter of lesions was measured at 3 days after inoculation. In both NtMKP1-AS lines, necrotic lesions were approximately $50 \%$ smaller than in the control line (Student's $t$ test, $P<0.01$ ) and chlorosis around lesions was pronounced (Fig. 4A and B). Furthermore, the NtMKP1 AS12 line showed reduction of $28 \%(P<0.01)$ of the lesion size compared with controls after inoculation with a conidial suspension (Fig. 4C). Hence, silencing of NtMKPl suppressed penetration and colonization by $B$. cinerea.

Then we assessed the effect of NtMPK1 transcript suppression upon susceptibility against TMV, a typical biotrophic pathogen. TMV was inoculated into detached leaves of
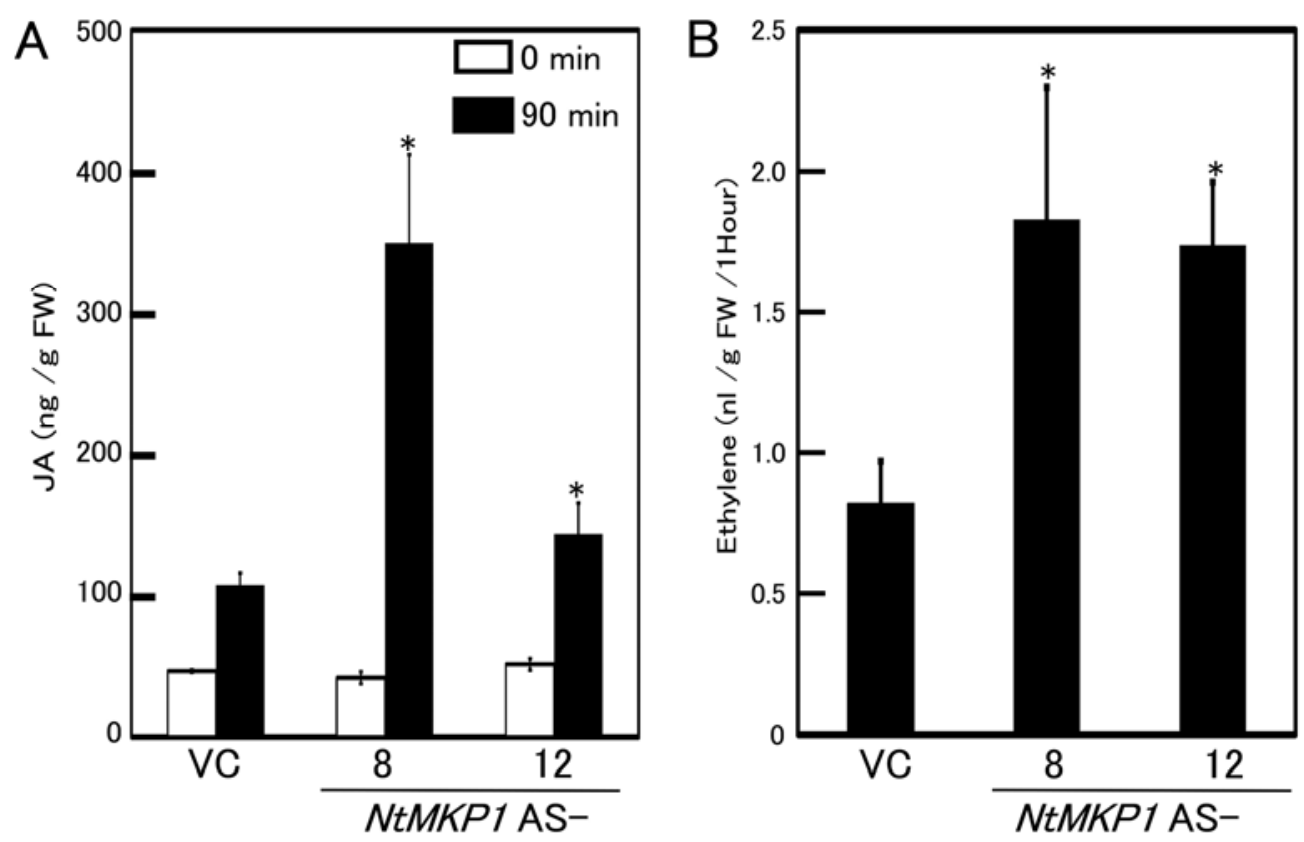

Fig. 2. Levels of jasmonic acid (JA) and ethylene (ET) in NtMKP1-suppressed tobacco plants after wounding. A, Levels of JA in vector control (VC) and NtMKP1-AS lines were measured at 90 min before (white bars) and after (black bars) wounding. The amount of JA is expressed as nanograms per gram fresh weight ( $\left.\mathrm{ng} \mathrm{g}^{-1} \mathrm{FW}\right)$. B, ET emission from leaves of VC and NtMKP1-AS lines after wounding. Leaves were cut into small pieces and incubated in a sealed vial for $4 \mathrm{~h}$. For A and B, data represent the means \pm standard error of three independent plants. Significant differences analyzed by Mann-Whitney's $u$ test between the control and transgenic lines are indicated $(*=P<0.05)$. 
NtMKP1-AS and the control lines and diameters of hypersensitive-respone lesions were measured at 5 days after inoculation. The lesion sizes were not significantly different between NtMKP1-AS and the control lines (Supplementary Fig. S3).

We next evaluated the effect of NtMKPl suppression on survival rate and growth of the lepidopteran herbivores, cabbage moth (Mamestra brassicae) and tobacco cutworm (Spodoptera litura). The survival rate of first-instar larvae of $M$. brassicae fed on the NtMKP1 AS8 and AS12 lines was markedly lower than those fed on control line at 3 days after inoculation (Mann-Whitney's $u$ test, $P<0.05$ and 0.01 , respectively) (Fig. $5 \mathrm{~A})$. The effect of NtMKP1 suppression on the survival rate of $M$. brassicae larvae was also assessed using third-instar larvae. The survival rate of third-instar larvae fed on both NtMKP1AS lines was decreased significantly compared with that on the control at 3 days after inoculation (nominal logistic regression analysis, $P<0.05$ ) (Fig. 5B). Then, the effect of $N t M K P I$ suppression on the survival rate of another lepidopteran herbivore, $S$. litura, was assayed. The survival rate of larvae fed on leaves of NtMKPI AS8 and AS12 lines was significantly lower than that on the control line at 2 days after inoculation (MannWhitney's $u$ test, $P<0.01$ and 0.05 , respectively) (Fig. 5C).

\section{DISCUSSION}

In this study, suppression of NtMKPl in transgenic tobacco resulted in enhanced the activation of tobacco MAPK WIPK and SIPK and production of both JA and ET. Furthermore,
NtMKP1-suppressed plants exhibited enhanced resistance against the necrotrophic pathogen $B$. cinerea and lepidopteran herbivores $M$. brassicae and $S$. litura. Our previous reports (Seo et al. 2007; Yamakawa et al. 2004), that overexpression of NtMKP1 represses wound-induced activation of WIPK and SIPK, suggested that NtMKP1 can inactivate these MAPK in vivo. Here, we revealed that NtMKP1 negatively regulates wound-induced activation of tobacco MAPK and using NtMKP1-suppressed transgenic plants.

Similarly, Katou and associates (2007) reported enhanced activation of OsMPK3 and OsMKP6, which are the orthologs of WIPK and SIPK, respectively, in the OsMKPI loss-of-function mutant (osmkpl). OsMPK3 and OsMPK6 were constitutively activated in $o s m k p l$ rice plants, whereas the activation of WIPK and SIPK was not constitutive in NtMKPl-suppressed tobacco plants (Fig. 1B) as well as in the AtMKPl loss-offunction mutant (atmkpl) (Ulm et al. 2002). Growth of the NtMKP1-AS lines was indistinguishable from wild-type plants under normal conditions, whereas osmkpl exhibited a semidwarf phenotype (Katou et al. 1997). Mutant atmkpl in a Columbia ( $\mathrm{Col}$ ) background also exhibited a constitutive stress response phenotype, causing aberrant growth and development (Bartels et al. 2009). However, atmkpl in a Wassilewskija (Ws) ecotype background does not exhibit constitutive defense or delayed growth, as observed in NtMKPl-AS tobacco plants (Ulm et al. 2001). Bartels and associates (2009) concluded that the difference in the atmkpl phenotype between the Ws and Col ecotype backgrounds was largely caused by the Col-spe-
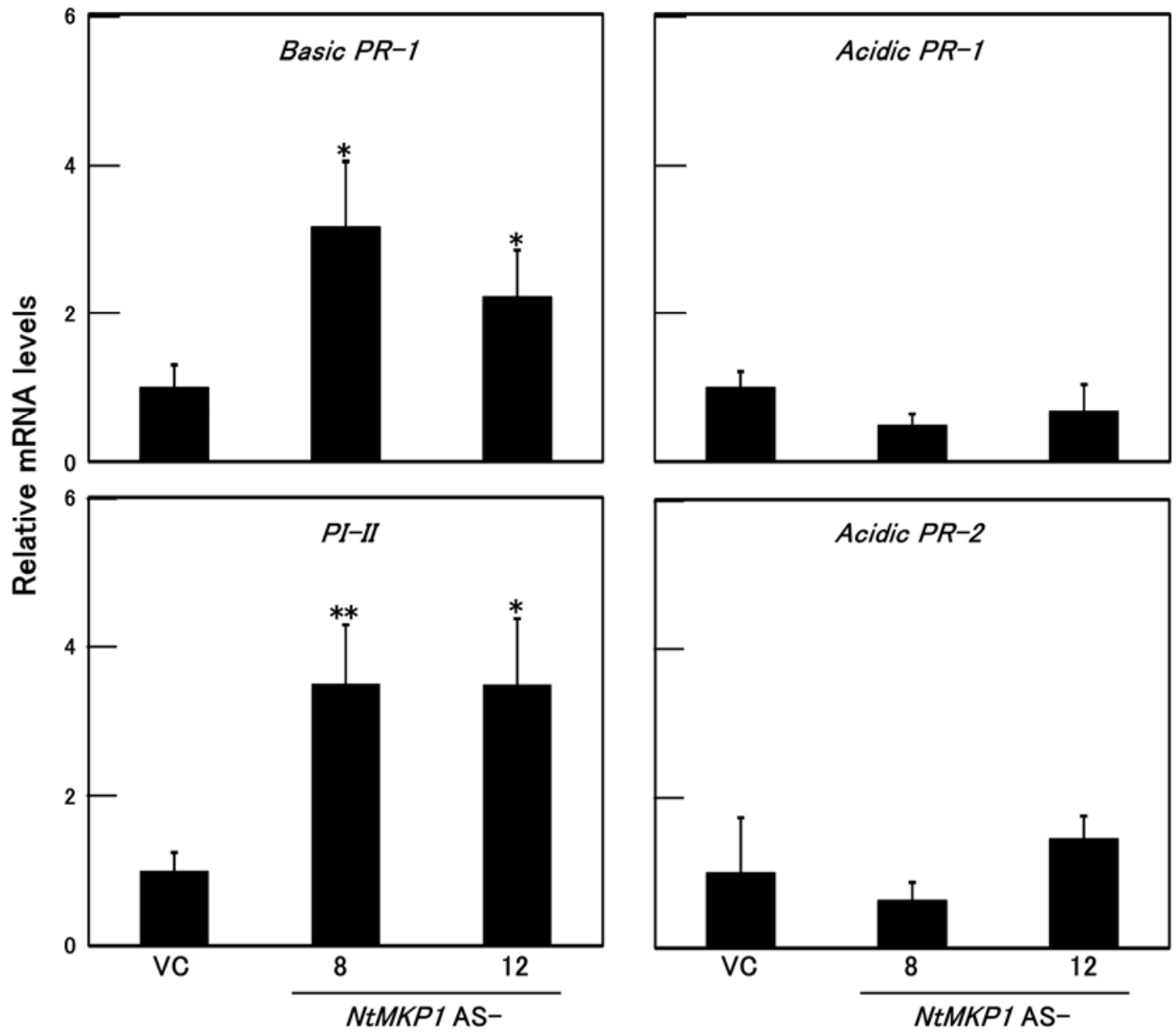

Fig. 3. Expression of defense-related genes in wounded NtMKP1-suppressed tobacco plants. Leaves of vector control (VC) and NtMKP1-AS lines were harvested at $24 \mathrm{~h}$ after wounding. Transcript levels of each gene were determined by quantitative real-time polymerase chain reaction, normalized by that of actin as an internal standard, and shown as a value relative to that of VC. Data represent the means \pm standard error of three independent plants for VC and NtMKP1-AS lines. Significant differences analyzed by Student's $t$ test between the control and transgenic lines are indicated ( $* *$ and $*=P<0.01$ and 0.05 , respectively). 


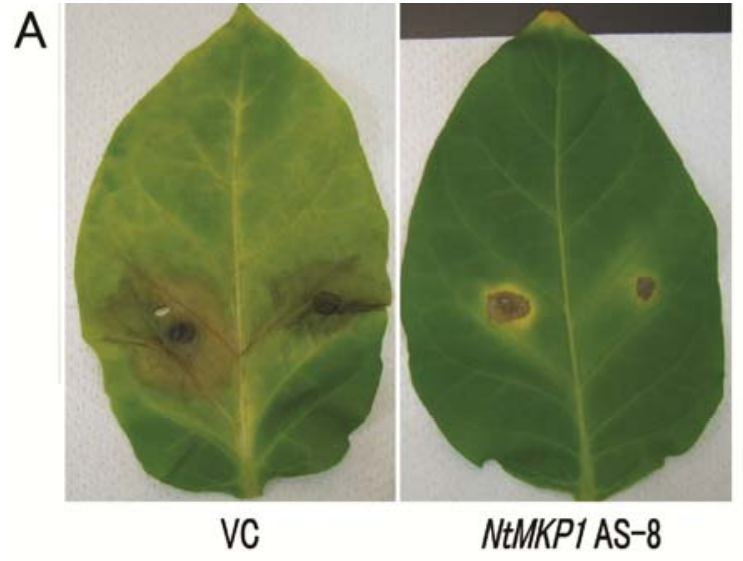

B

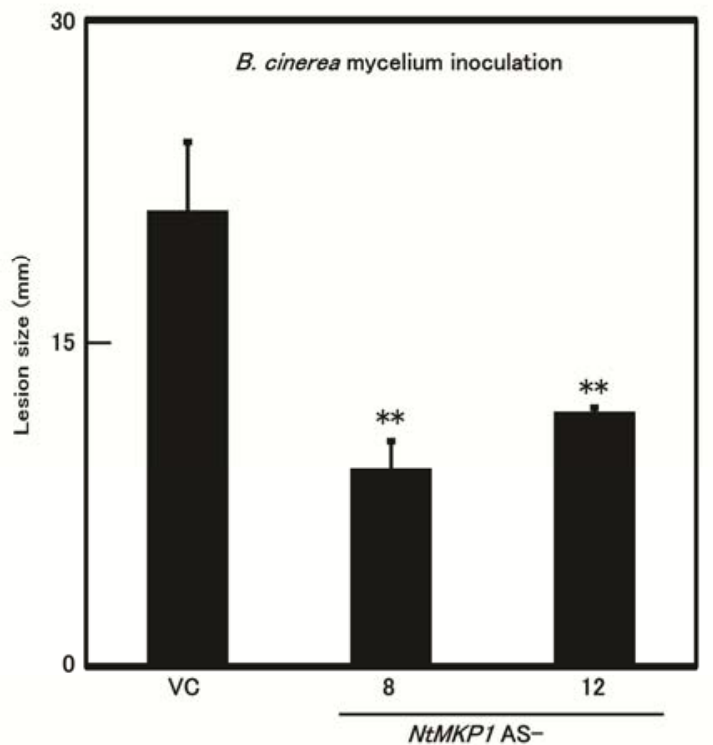

C

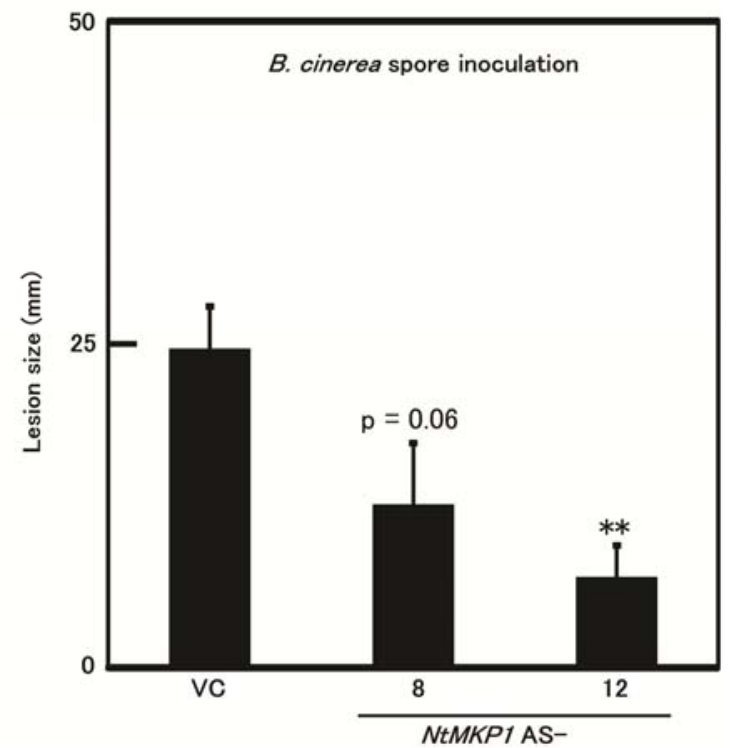

Fig. 4. Suppression of NtMKPI enhances the resistance of tobacco against Botrytis cinerea. A and $\mathbf{B}$, Leaves of vector control (VC) and NtMKP1-AS lines were inoculated with a culture of $B$. cinerea mycelium. A, Leaves showed necrosis lesions at 3 days after inoculation. B, Diameter of necrotic lesions was measured and means \pm standard error (SE) are presented. C, Leaves of VC and NtMKPI-AS lines were inoculated with conidia of $B$. cinerea. After 3 days, the diameter of necrotic lesions was measured. For B and C, data represent the means \pm SE of six independent experiments. Significant differences analyzed by Student's $t$ test between the control and transgenic lines are indicated. $(* *=P<0.01)$ cific toll interleukin 1 receptor nucleotide-binding leucine-rich repeat receptor-like protein SNC1. Thus, the abnormal phenotype observed in atmkpl in a $\mathrm{Col}$ background seems rather specific.

Suppression of NtMKPI resulted in enhanced JA and ET accumulation upon wounding (Fig. 2), whereas both WIPK- or $S I P K$-silenced and NtMKPl-overexpressed lines exhibited reduced JA and ET production in response to wounding (Seo et al. 2007). Furthermore, expression of the JA- or ET-inducible genes basic PR-1 and PI-II was significantly enhanced by wounding but the SA-inducible genes acidic $P R-1$ and acidic $P R-2$ was not significantly altered in NtMKPl-AS lines (Fig. $3)$. These lines of evidence indicate that NtMKP1 negatively regulates the production of JA and ET and the expression of JA- or ET-inducible genes through suppression of MAPK WIPK and SIPK.

Plant resistance to necrotrophic pathogens is mediated by JA and ET signaling, a process also involved in plant responses to wounding (Rossi et al. 2011; Spoel et al. 2007). Enhanced resistance against both necrotrophic pathogens and lepidopteran herbivores in NtMKPl-suppressed tobacco plants (Figs. 4 and 5) might be a result of enhanced activation of WIPK and SIPK leading to JA or ET accumulation. Previous studies indicated that Arabidopsis and N. benthamiana MAPK, MPK3/MPK6 or SIPK, are activated upon inoculation with $B$. cinerea and contribute to resistance against $B$. cinerea (Asai and Yoshioka 2009; Ren et al. 2008). Thus, enhanced resistance against $B$. cinerea in the NtMKP1-suppressed tobacco plants might be due to the enhanced activation of MAPK upon infection. Although MPK3 activity was enhanced in the Arabidopsis mutant lacking MKP2 after infection of $B$. cinerea, the silencing of MKP2 produced accelerated sensitivity to $B$. cinerea (Lumbreras et al. 2010). The function of MKP2 on MAPK cascades is likely to be different from NtMKP1. However, because the interaction between MKP and MAPK is capable of modulating other processes or factors, future studies will be necessary to define the exact molecular mechanism.

Some studies reported that suppression or overexpression of genes that regulate JA or ET production or signaling resulted in enhanced multiple resistances but constitutively activated defense responses often resulted in growth retardation and miniaturization (Ellis and Turner 2001; Qiu et al. 2012; Qu et al. 2010). In contrast, the suppression of NtMKPl confers multiple resistances against both necrotrophic pathogen and herbivorous pests without deleterious consequences. Schweighofer and associates (2007) reported previously the pronounced activation of AtMPK6 and AtMPK4 and enhanced resistance against both B. cinerea and Tetranychus urticae (spider mite) in an Arabidopsis mutant lacking AP2C, which was the PP2C-type phosphatase. MKP negatively regulate activated MAPK; therefore, suppression of MKP may hardly affect plant growth and developments under normal growth conditions, in which MAPK are not activated, whereas activation of MAPK upon stimulation are enhanced in the NtMPK1-suppressed plants. In fact, neither the levels of MAPK activity nor accumulation of JA was different between NtMKPl-AS lines and control plants before wounding (Figs. 1 and 2).

Recently, several types of gene manipulation technique that enable the production of plants with targeted gene knockdown or knockout without any artificial recombinant DNA sequence, such as RNA-mediated DNA methylation, ZEN nuclease, and TALLEN nuclease system, have been highlighted as "new plant breeding techniques" (Lusser et al 2012). Thus, genetic knockdown or knockout of negative regulators of defense responses could be a promising way for the production of pathogen or herbivore resistant crops without transgenes, and NtMKP1 may be a possible target for such interventions. 


\section{MATERIALS AND METHODS}

Plasmid construction and tobacco transformation.

A binary vector expressing $N t M K P 1$ antisense RNA under the control of an enhanced $35 \mathrm{~S}$ promoter was constructed as described by Yamakawa and associates (2004), except for the orientation of the NtMKPl open reading frame. The construct was used to transform tobacco ( $N$. tabacum 'Samsun $N N$ ') by the leaf disc co-cultivation method with Agrobacterium tumefaciens LBA4404 (Horsch et al. 1985).

\section{Plant materials and plant growth conditions.}

Kanamycin-resistant seedlings from the transgenic lines were transferred to pots containing vermiculite and grown in a chamber maintained at $25^{\circ} \mathrm{C}$ with $16 \mathrm{~h}$ of light $(120 \mu \mathrm{mol} \mathrm{mmol}$ $\left.\mathrm{m}^{-2} \mathrm{~s}^{-1}\right)$. For each assay and analysis, 2-month-old tobacco plants were used.

\section{Immunocomplex kinase assays.}

MBP kinase activity of SIPK and WIPK was measured as described previously (Seo et al. 1999).

\section{Phytohormone measurements.}

Extraction and quantification of JA were performed essentially as described previously (Baldwin et al. 1997), except that $\left[{ }^{2} \mathrm{H}_{2}\right]( \pm)-J A$ was used as an internal standard (Nojiri et al. 1992). Quantification of SA and SAG was performed as described previously (Seo et al. 1995). Measurement of ET was performed as described previously (Ohtsubo et al. 1999).

RNA extraction and quantitative real-time PCR analyses.

Total RNA was extracted by using TRIzol reagent in accordance with the manufacturer's instructions (Invitrogen, Carlsbad, CA, U.S.A.).

First-strand cDNA was synthesized using an iScript cDNA synthesis kit (Bio-Rad, Hercules, CA, U.S.A.). Quantitative real-time PCR was conducted using iQ SYBR Green Supermix (Bio-Rad) and the following primers; for NtMKP1-open reading frame, 5'-CCTCACTCTCACCTTCAACCTCAG-3' and 5'-GTATGCCAAGTCGGACCAATTAGAAG-3'; for SIPK, 5' CCACGGTGGCAGGTTCATTC- $3^{\prime}$ and 5'-CAGAACAAAC GATGCCGTAAGC-3'; for WIPK, 5'-CCAAGTATCGTCCTC CTATTATG-3' and 5'-TCACGGAGAGTCCTCTTAGC-3'; for $P I$ - II, 5'-ATGCTAAACTCTGTAATGTGGGC-3' and 5'-CATT AAATTCTTGGGCATGTCCTTG-3'; for Basic PR-1, 5'-TTT TGGTGGTATTATGGAGGTGTG- $3^{\prime}$ and $5^{\prime}$-ACAATTAACTG CCGTTGACTCATC-3'; for Acidic PR-1, 5'-GTCCATACTAA TTGAAACGACCTAC-3' and 5'-CCACTTCAGAGGATTAC ATATATAGTAC-3'; for Acidic PR-2, 5'-TGAGTAATAAGGA

Fig. 5. $N t M K P 1$-suppressed tobacco plants exhibit increased resistance against lepidopteran herbivores. A, Ten first-instar larvae of Mamestra brassicae were placed on one leaf detached from one tobacco plant of the vector control (VC) or NtMKP1-AS lines, and allowed to feed. After 3 days after inoculation (DAI), the number of surviving larvae on each leaf was counted. Data represent the means \pm standard error (SE) of 10 independent experiments. B, A third-instar larva of $M$. brassicae was placed on one detached leaf of VC and NtMKP1-AS lines. After 3 DAI, the survival rate of 16 larvae was determined. Values with different letters are significantly different by nominal logistic regression analysis $(P<$ 0.05). C, Ten first-instar larvae of Spodoptera litura were placed on one leaf excised from one tobacco plant of the VC and NtMKPl-AS lines, and allowed to feed. After 2 DAI, the number of surviving larvae was counted. Data represent the means \pm SE of 10 independent experiments. For $\mathrm{A}$ and $\mathrm{C}$, significant differences analyzed using Mann-Whitney's $u$ test between the control and transgenic lines are indicated $\left(^{*}\right.$ and $* *=P<0.05$ and 0.01 , respectively).
GAAGTAGAACTG-3' and 5'-AATATCAAAGTGAAACCAG AGTTA-3'; and for actin, 5'-GGGTTTGCTGGAGATGATG

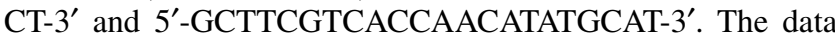
were normalized to the expression of the tobacco actin mRNA.

Bioassay for B. cinerea and TMV.

The isolate (National Institute of Agrobiological Sciences GeneBank, MAFF number 306658) of B. cinerea Persoon was
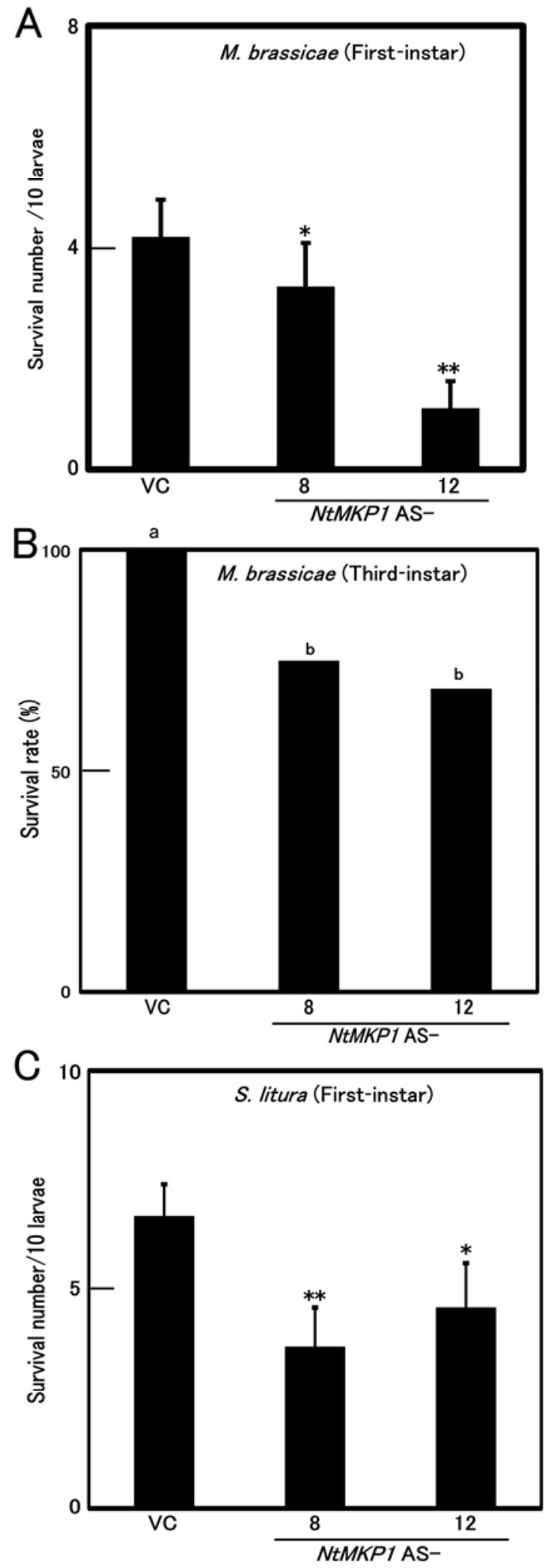

Vol. 26, No. 6, 2013 / 673 
grown on potato dextrose agar under UV light and darkness of 12 and $12 \mathrm{~h}$, respectively, for 3 days and then under only a dark regime for 2 weeks. The mycelia disk $(10 \mathrm{~mm}$ in diameter) was placed on the surface of detached tobacco leaves. Droplets $(20 \mu \mathrm{l})$ of spore suspension $\left(10^{6}\right.$ spores $\left.\mathrm{ml}^{-1}\right)$ with distilled water were inoculated on tobacco leaves. Inoculated leaves were kept at $100 \%$ humidity and $25^{\circ} \mathrm{C}$, and the diameter of lesion size was measured at 3 days after inoculation. For each experiment, three leaves from three tobacco plants from each line were used and the same experiments were repeated six times.

The leaf surfaces of intact plants were rubbed gently with Carborundum and TMV-OM at $0.4 \mu \mathrm{g} \mathrm{ml}^{-1}$ suspended in 10 $\mathrm{mM}$ phosphate buffer ( $\mathrm{pH}$ 7.0). After rinsing under a gentle stream of tap water, inoculated plants were placed in a chamber maintained at $20^{\circ} \mathrm{C}$. At 5 days after inoculation, the diameter of local necrotic lesions was measured.

\section{Bioassay for lepidopteran herbivores larvae.}

Fully expanded upper leaves were excised from approximately 6-week-old tobacco plants and the petioles were inserted into plastic bags containing cotton with distilled water just before inoculation. Ten newly hatched larvae of both $M$. brassicae and S. litura or one third-instar larva of M. brassicae was placed on a tobacco leaf in a plastic case at $25 \pm 1^{\circ} \mathrm{C}$. Ten replicates were done for first-instar larvae and sixteen replicates for third-instar larvae.

\section{ACKNOWLEDGMENTS}

This work was supported by the Japanese Program for the Promotion of Basic and Applied Researches for Innovation in Bio-oriented Industry (BRAIN).

\section{LITERATURE CITED}

Anderson, J. C., Bartels, S., Besteiro, M. A. G., Shahollari, B., Ulm, R., and Peck, S. C. 2011. Arabidopsis MAP kinase phosphatase 1 (AtMKP1) negatively regulates MPK6-mediated PAMP responses and resistance against bacteria. Plant J. 67:258-268.

Asai, S., and Yoshioka, H. 2009. Nitric oxide as a partner of reactive oxygen species participates in disease resistance to necrotrophic pathogen Botrytis cinerea in Nicotiana benthamiana. Mol. Plant-Microbe Interact. 22:619-629.

Baldwin, I. T., Zhang, Z. P., Diab, N., Ohnmeiss, T. E., McCloud, E. S., Lynds, G. Y., and Schmeiz, E. A. 1997. Quantification, correlations and manipulations of wound-induced changes in jasmonic acid and nicotine in Nicotiana sylvestris. Planta 201:397-404.

Bartels, S., Anderson, J. C., Besteiro, M. A. G., Carreri, A., Hirt, H., Buchala, A., Metraux, J.-P., Peck, S. C., and Ulm, R. 2009. MAP KINASE PHOSPHATASE 1 and PROTEIN TYROSINE PHOSPHATASE 1 are repressors of salicylic acid synthesis and SNC1-mediated responses in Arabidopsis. Plant Cell 21:2884-2897.

Bostock, R. M. 1999. Signal conflicts and synergies in induced resistance to multiple attackers. Physiol. Mol. Plant Pathol. 55:99-109.

Brederode, F. T., Linthorst, H. J. M., and Bol, J. F. 1991. Differential induction of acquired resistance and PR gene expression in tobacco by virus infection, ethephon treatment, UV light and wounding. Plant Mol. Biol. 17:1117-1125.

Browse, J. 2009. Jasmonate passes muster: A receptor and targets for the defense hormone. Annu. Rev. Plant Biol. 60:183-205.

Camps, M., Nichols, A., and Arkinstall, S. 2000. Dual specificity Phosphatases: A gene family for control of MAP kinase function. FASEB (Fed. Am. Soc. Exp. Biol.) J. 14:6-16.

Diaz, J., ten Have, A., and van Kan, J. A. 2002. The role of ethylene and wound signaling in resistance of tomato to Botrytis cinerea. Plant Physiol. 129:1341-1351.

Ellis, C., and Turner, J. G. 2001. The Arabidopsis mutant cev1 has constitutively active jasmonate and ethylene signal pathways and enhanced resistance to pathogens. Plant Cell 13:1025-1033.

Glazebrook, J. 2005. Contrasting mechanisms of defense against biotrophic and necrotrophic pathogens. Annu. Rev. Phytopathol. 43:205-227.
Gupta, R., Huang, Y., Kieber, J., and Luan, S. 1998. Identification of a dual-specificity protein phosphatase that inactivates a MAP kinase from Arabidopsis. Plant J. 16:581-589.

Hind, S. R., Pulliam, S. E., Veronese, P., Shantharaj, D., Nazir, A., Jacobs, N. S., and Stratmann, J. W. 2011. The COP9 signalosome controls jasmonic acid synthesis and plant responses to herbivory and pathogens. Plant J. 65:480-491.

Horsch, R. B., Fry, J. E., Hoffmann, N. L., Eichholtz, D., Rogers, S. G., and Fraley, R. T. 1985. A simple and general method for transferring genes into plants. Science 227:1229-1231.

Howe, G. A., and Jander, G. 2008. Plant immunity to insect herbivores. Annu. Rev. Plant Biol. 59:41-66.

Kandoth, P. K., Ranf, S., Pancholi, S. S., Jayanty, S., Walla, M. D., Miller, W., Howe, G. A., Lincoln, D. E., and Stratmann, J. W. 2007. Tomato MAPK LeMKP1, LeMKP2, and LeMKP3 function in the systemin-mediated defense response against herbivorous insects. Proc. Natl. Acad. Sci. U.S.A. 104:12205-12210.

Katou, S., Karita, E., Yamakawa, H., Seo, S., Mitsuhara, I., Kuchitsu, K., and Ohashi, Y. 2005. Catalytic activation of the plant MAPK phosphatase NtMKP1 by its physiological substrate salicylic acid-induced protein kinase but not by calmodulins. J. Biol. Chem. 280:39569-39581.

Katou, S., Kuroda, K., Seo, S., Yanagawa, Y., Tsuge, T., Yamazaki, M., Miyao, A., Hirochika, H., and Ohashi, Y. 2007. A calmodulin-binding mitogen-activated protein kinase phosphatase is induced by wounding and regulates the activities of stress-related mitogen-activated protein kinases in rice. Plant Cell Physiol. 48:332-344.

Kerk, D., Bulgrien, J., Smith, D. W., Barsam, B., Veretnik, S., and Gribskov, M. 2002. The complement of protein phosphatase catalytic subunits encoded in the genome of Arabidopsis. Plant Physiol. 129:908-925.

Kobayashi, M., Seo, S., Hirai, K., Yamamoto-Katou, A., Katou, S., Seto, H., Meshi, T., Mitsuhara, I., and Ohashi, Y. 2010. Silencing of WIPK and SIPK mitogen-activated protein kinases reduces Tobacco mosaic virus accumulation but permits systemic viral movement in tobacco possessing the $N$ resistance gene. Mol. Plant-Microbe Interact. 23:10321041

Koornneef, A., and Pieterse, C. M. 2008. Cross talk in defense signaling. Plant Physiol. 146:839-844.

Lee, J. S., and Ellis, B. E. 2007. Arabidopsis MAPK phosphatase 2 (MKP2) positively regulates oxidative stress tolerance and inactivates the MPK3 and MPK6 MAPK. J. Biol. Chem. 282:25020-25029.

Lee, J. S., Wang, S., Sritubtim, S., Chen, J. G., and Ellis, B. E. 2009. Arabidopsis mitogen-activated protein kinase MPK12 interacts with the MAPK phosphatase IBR5 and regulates auxin signaling. Plant J. 57:975-985.

Lumbreras, V., Vilela, B., Lrar, S., Sole, M., Capellades, M., Valls, M., Coca, M., and Pages, M. 2010. MAPK phosphatase MKP2 mediates disease responses in Arabidopsis and functionally interacts with MPK3 and MPK6. Plant J. 63:1017-1030.

Lusser, M., Parisi, C., Plan, D., and Rodriguez-Cerezo, E. 2012. Deployment of new biotechnologies in plant breeding. Nat. Biotechnol. 30:231-239.

MAPK Group 2002. Mitogen-activated protein kinase cascades in plants: A new nomenclature. Trends Plant Sci. 7:301-308

Meldau, S., Wu, J., and Baldwin, I. T. 2009. Silencing two herbivory-activated MAP kinases, SIPK and WIPK, does not increase Nicotiana attenuata's susceptibility to herbivores in the glasshouse and in nature. New Phytol. 181:161-173.

Monroe-Augustus, M., Zolman, B. K., and Bartel, B. 2003. IBR5, a dualspecificity phosphatase-like protein modulating auxin and abscisic acid responsiveness in Arabidopsis. Plant Cell 15:2979-2991.

Nakagami, H., Pitzschke, A., and Hirt, H. 2005. Emerging MAP kinase pathways in plant stress signaling. Trends Plant Sci. 10:339-346.

Naoi, K., and Hashimoto, T. 2004. A semidominant mutation in an Arabidopsis mitogen-activated protein kinase phosphatase-like gene compromises cortical microtubule organization. Plant Cell 16:1841-1853.

Niki, T., Mitsuhara, I., Seo, S., Ohtsubo, N., and Ohashi, Y. 1998. Antagonistic effect of salicylic acid and jasmonic acid on the expression of pathogenesis-related (PR) protein genes in wounded mature tobacco leaves. Plant Cell Physiol. 39:500-507.

Nojiri, H., Yamane, H., Seto, H., Yamaguchi, I., Murofushi, N., Yoshihara, T., and Shibaoka, H. 1992. Qualitative and quantitative analysis of endogenous jasmonic acid in bulbing and non-bulbing onion plants. Plant Cell Physiol. 33:1225-1231.

Ohtsubo, N., Mitsuhara, I., Koga, M., Seo, S., and Ohashi, Y. 1999. Ethylene promotes the necrotic lesion formation and basic PR gene expression in TMV-infected tobacco. Plant Cell Physiol. 40:808-817.

Pitzschke, A., Schikora, A., and Hirt, H. 2009. MAPK cascade signaling networks in plant defense. Plant Biol. 12:421-426.

Qiu, Y., Xi, J., Du, L., Suttle, J. C., and Poovaiah, B. W. 2012. Coupling calcium/calmodulin-mediated signaling and herbivore-induced plant 
response through calmodulin-binding transcription factor AtSR1/ CAMYA3. Plant Mol. Biol. 79:89-99.

Qu, N., Gan, W., Bi, D., Xia, S., Li, X., and Zhang, Y. 2010. Two BTB proteins function redundantly as negative regulators of defense against pathogens in Arabidopsis. Botany 88:953-960.

Ren, D., Liu, Y., Yang, K. Y., Han, L., Mao, G., Glazebrook, J., and Zhang, S. 2008. A fungal-responsive MAPK cascade regulates phytoalexin biosynthesis in Arabidopsis. Proc. Natl. Acad. Sci. U.S.A. 105:56385643.

Rossi, F. R., Garriz, A. Marina, M., Romero, F. M., Gonzalez, M. E., Collado, I. G., and Pieckenstain, F. L. 2011. The sesquiterpene botrydial produced by Botrytis cinerea induces the hypersensitive response on plant tissues and its action is modulated by salicylic acid and jasmonic acid signaling. Mol. Plant-Microbe Interact. 24:888-896.

Schweighofer, A., Kazanaviciute, V., Scheikl, E., Teige, M., Doczi, R. Hirt, H., Schewanninger, M., Kant, M., Schuurink, R., Mauch, F. Buchala, A., Cardinala, F., and Meskiene, I. 2007. The PP2C-type phosphatase AP2C1, which negatively regulates MPK4 and MKP6, modulates innate immunity, jasmonic acid, and ethylene levels in Arabidopsis. Plant Cell 19:2213-2224.

Seo, S., Okamoto, M., Seto, H., Ishizuka, K., Sano, H., and Ohashi, Y. 1995. Tobacco MAP kinase: A possible mediator in wound signal transduction pathways. Science 207:1988-1992.

Seo, S., Sano, H., and Ohashi Y. 1999. Jasmonate-based wound signal transduction requires activation of WIPK, a tobacco mitogen-activated protein kinase. Plant Cell 11:289-298.

Seo, S., Katou, S., Seto, H., Gomi, K., and Ohashi, Y. 2007. The mitogenactivated protein kinases WIPK and SIPK regulate the levels of jasmonic and salicylic acids in wounded tobacco plants. Plant $\mathrm{J}$. 49:899-909.
Spoel, S., Johnson, J. S., and Dong, X. 2007. Regulation of tradeoffs between plant defenses against pathogens with different lifestyles. Proc. Natl. Acad. Sci. U.S.A. 104:18842-18847.

Turner, J. G., Ellis, C., and Devoto, A. 2002. The jasmonate signal pathway. Plant Cell 14:153-164.

Ulm, R., Revenkova, E., di Sansebastiano, G.-P., Bechtold, N., and Paszkowski, J. 2001. Mitogen-activated protein kinase phosphatase is required for genotoxic stress relief in Arabidopsis. Genes Dev. 15:699709.

Ulm, R., Ichimura, K., Mizoguchi, T., Peck, S. C., Zhu, T., Wang, X., Shinozaki, K., and Paszkowski, J. 2002. Distinct regulation of salinity and genotoxic stress response by Arabidopsis MAP kinase phosphatase 1. EMBO (Eur. Mol. Biol. Organ.) J. 21:6483-6493.

Walia, A., Lee, J. S., Wasteneys, G., and Ellis, B. 2009. Arabidopsis mitogen-activated protein kinase MPK18 mediates cortical microtubule functions in plant cells. Plant J. 59:565-575

Wu, J., Hettenhausen, C., Meldau, S., and Baldwin, T. 2007. Herbivory rapidly activates MAPK signaling in attacked and unattacked leaf regions but not between leaves of Nicotiana attenuate. Plant Cell 19:1096-1122.

Yamakawa, H., Katou, S., Seo, S., Mitsuhara, I., Kamada, H., and Ohashi, Y. 2004. Plant MAPK phosphatase interacts with calmodulins. J. Biol. Chem. 279:928-936.

Zhang, S., and Klessig, D.F. 1997. Salicylic acid activates a 48-kD MAP kinase in tobacco. Plant Cell 9:809-824.

Zhang, S., and Klessig, D.F. 2001. MAPK cascades in plant defense signaling. Trends Plant Sci. 6:520-527.

Zhang, S., and Liu, Y. 2001. Activation of salicylic acid-induced protein kinase, a mitogen-activated protein kinase, induces multiple defense responses in tobacco. Plant Cell 13:1877-1889. 